\title{
Searching for Galactic sources in the Swift GRB catalog
}

\author{
J.C.Tello*, ${ }^{1}$ A.J. Castro-Tirado, ${ }^{1}$ J. Gorosabel, ${ }^{1}$ P.Veres,${ }^{2,3}$ Z. Bagoly, ${ }^{2}$ D. \\ Pérez-Ramírez ${ }^{4}$ and S.Guziy ${ }^{5} \dagger$ \\ ${ }^{1}$ Instituto de Astrofísica de Andalucía (IAA-CSIC) \\ Granada, Spain \\ ${ }^{2}$ Eötvös University \\ Budapest, Hungary \\ ${ }^{3}$ Bolyai Military University \\ Budapest, Hungary \\ ${ }^{4}$ Departamento de Física, Universidad de Jaén \\ Jaén, Spain \\ ${ }^{5}$ Nikolaev National University \\ Nikolaev, Ukraine \\ E-mail: jtello@iaa.es
}

\begin{abstract}
Since the early 90s Gamma Ray Bursts have been accepted to be of extragalactic origin thanks to the isotropic distribution observed by BATSE and the redshifts observed in some of their optical or infrared counterparts. Nevertheless, there have been a few cases that upon further examination have turned out to be of Galactic origin. In this work we will conduct several statistical analyses to estimate the most probable degree of contamination by Galactic sources of different samples taken from the Swift GRB catalog. This work details the proposed methodology.
\end{abstract}

Gamma-Ray Bursts 2012 Conference-GRB2012,

May 07-11, 2012

Munich, Germany

\footnotetext{
* Speaker.

${ }^{\dagger}$ This research has been made possible thanks to the support of the Spanish MICINN program AYA 2009-14000C03-01.
} 


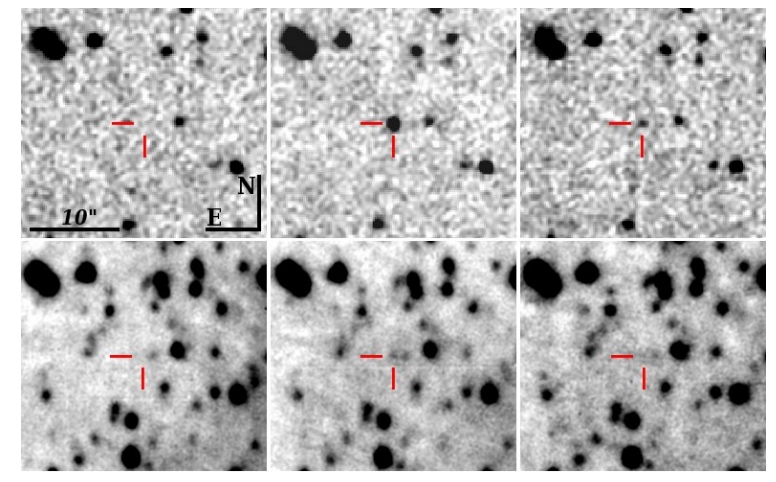

Figure 1: Flaring activity of GRB 070610/Swift J1955+2604, a Galactic source mimicking a 8s longduration GRB which is proposed to be either an evolved magnetar or an ultrashort low-mass X-ray binary. $[1][2][3]$

\section{Introduction}

Gamma Ray Bursts (GRBs) are now generally accepted to be of extragalactic origin despite of early opposition due to their unparalleled energy emission. This change of paradigm was first suggested by the isotropy of the very large BATSE GRB catalog, or more importantly the lack of anisotropy with respect to the Galactic Plane. However, this fact by itself was not enough and theories coherent with this isotropy arose that were compatible with sources within the Milky Way, although unlikely.

The observation of GRB970508 which was the first burst whose redshift was recorded allowed very definitive proof that GRBs in general were of extragalactic origin, and also the most energetic events in the Universe besides The Big Bang.

However not all GRBs have a detected redshift, and more importantly, upon thorough examination several events originally cataloged as GRBs have been pointed out to be more likely of a different, Galactic, nature (See figure 1). This has lead us to consider that it could be useful to actively look for other Galactic sources within GRB archives.

\section{Sample Selection}

We will select several subsamples of the Swift GRB catalog, using criteria based on publications that matches those of sources which were previously thought to be GRBs, but were in fact Galactic sources. Each of these subsamples will then be tested to determine if they deviate from isotropy. In order to quantify the degree of anisotropy with respect to the Galactic Plane we will use indicators like the mean dipolar moment of the samples' latitudes, among others, and compare these to simulated samples.

\section{Isotropic distributions}

We will consider the probability distribution of the anisotropy indicators of a randomly generated isotropic sample. To ensure that the sample is generated isotropically we will first verify that the density of sources per solid angle is constant within statistical fluctuations (See Figure 2). We 


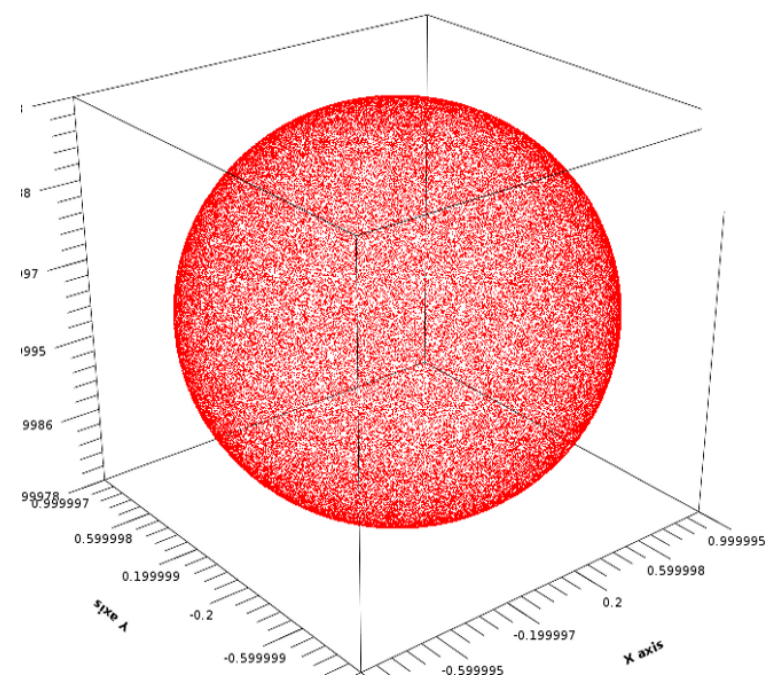

Figure 2: Millions of randomly generated homogeneous points on a sphere.

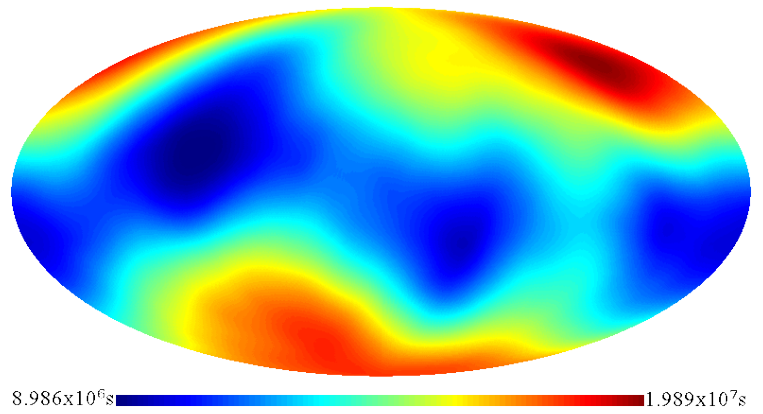

Figure 3: Exposure map of Swift's BAT instrument in Galactic coordinates. Red denotes higher exposure, where blue denotes lower exposure.

will then compare the indicators of our samples to see if they deviate with statistical significance from the isotropic distributions.

\section{Swift exposure map}

To take into account the anisotropy of Swift's exposure an exposure map has been created by integrating the exposure masks of the BAT instrument since its operational start [4]. This will be crucial as Swift's exposure of the sky is higher at the Galactic poles and less at the Galactic Plane (See Figure 3). We will weight the random isotropic sources using this map to cancel any bias that might be caused from the uneven observation of the sky.

\section{Contamination by Galactic sources}

In order to simulate the effect of sources from within the Milky Way, Galactic sources will be generated by weighting the randomly generated isotropic sources with the Schlegel et al. dust maps [6]. These dust maps were obtained by observing the infrared emission in the Milky Way with 


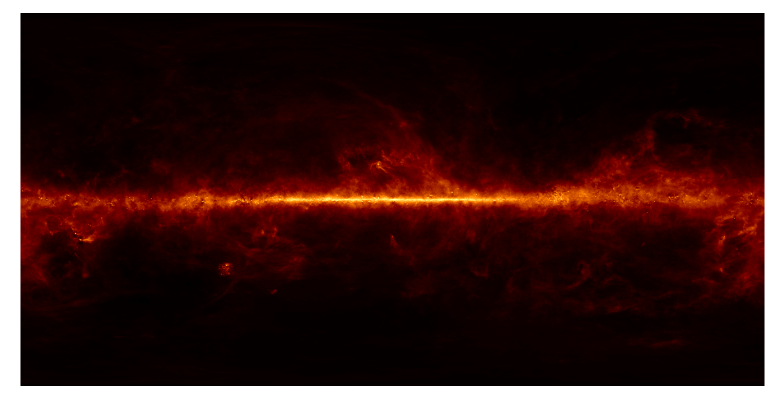

Figure 4: Galactic map of Infrared emission by interstellar dust [6].

satellites $C O B E$ and IRAS and carefully removing contributions by other factors like the zodiacal light and nearby stars (see Figure 4). These maps give a good estimation of the density of Galactic material which is why we believe it to be a good indicator of the density of Galactic sources.

\section{Future work}

We plan to publish an in-depth study regarding these analyses after having concluded thorough simulations that exclude possible statistical fluctuations and compensate for possible biases [7]. We also plan to expand the study to other catalogs such as BATSE's which will provide richer statistical samples but were not included in this study to ensure a proper study of instrumental bias.

Further work might include an attempt to correlate other features of probably sources, such as light curve parameters, to try and create a clear criteria to distinguish these contaminant sources from actual GRBs.

\section{References}

[1] A. J. Castro-Tirado, A. de Ugarte Postigo, J. Gorosabel, et al., Flares from a candidate Galactic magnetar suggest a missing link to dim isolated neutron stars, nat 455 (2008) 506 [astro-ph/0809.4231]

[2] N. Rea, P. G. Jonker, G. Nelemans, et al., The X-ray Quiescence of Swift J195509.6+261406 (GRB 070610): An Optical Bursting X-ray Binary?, ApJL 729 (2011) L21 [astro-ph/1101 . 3483]

[3] V. Simon, R. Hudec, A. J. Castro-Tirado, et al. Outburst and flares from the unique source SWIFT J1955+2614, MNRAS 422 (2012) 981

[4] P. Veres, Z. Bagoly, I. Horváth, et al.Directional Anisotropy of Swift Gamma-Ray Bursts, AIPC 1279 (2010) 457 [astro-ph/1012.1757]

[5] A. J. Castro Tirado, The WATCH experiment: 1000 days observing the X-ray universe., Ph.D. Thesis, University of Copenhagen, Copenhagen, Denmark, 1994,

[6] D. J. Schlegel, D. P. Finkbeiner, \& M. Davis,Maps of Dust Infrared Emission for Use in Estimation of Reddening and Cosmic Microwave Background Radiation Foregrounds, ApJ 525 (1998) 525 [astro-ph/9710327]

[7] J. C. Tello, A.J. Castro-Tirado, J. Gorosabel, et. al, In preparation, (2012) 